\title{
Estimation of the efficiency of the earthquake prediction on the basis of the analysis of ionospheric parameters
}

\author{
Vadim Bogdanov ${ }^{1, \star}$ and Aleksey Pavlov ${ }^{1, \star \star}$ \\ ${ }^{1}$ Institute of Cosmophysical Research and Radio Wave Propagation FEB RAS, Paratunka, Russia
}

\begin{abstract}
A method of the operational forecast of strong earthquakes is considered. Exceedance of the current values of ionospheric critical frequency $f_{0} F_{2}$ over the median values during the disturbed state of the magnetosphere is considered to be a precursor. The reliability, validity and the efficiency of the precursor were evaluated by A.A. Gusev's and G.M. Molchan's methods for the period of 2009-2016. Earthquakes with the magnitude of $M \geq 5.0$ occurring within the zone of precursor appearance determined by Dobrovol'skiy's radius were considered as forecasting earthquakes. It was shown that the precursor has the best predictive efficiency for the seismic events with the magnitude of $M \geq 6.5$.
\end{abstract}

\section{Introduction}

Based on the results of vertical radio sounding of ionospheric parameters, it was noted that for Kamchatka one of the most informative parameters is the critical frequency $f_{0} F_{2}$ of the regular $F_{2}$ layer [1]. On the eve of the seismic event in the dynamics of the behavior of the ionospheric parameters, the following anomalies are possible:

1. the shift of the maximum of the critical frequency $f_{0} F_{2}$ of the regular layer $F_{2}$ to the morning hours from midnight (UT) 3-5 days before the seismic event, which coincides with the results of other authors;

2. the excess of the current values of $f_{0} F_{2}$ over the median values, which is formed against the background of the perturbed magnetosphere, indicating that there is an additional ionization source in the $F_{2}$ layer associated with the electric field penetrating the ionospheric heights and resulting currents.

The complexity of observing ionospheric earthquake precursors is the difficulty in isolating lithospheric effects against the background of magnetospheric disturbances, this can only be done for strong events.

\footnotetext{
^e-mail: vbogd@ikir.ru

$\star \star$ e-mail: pavlov@ikir.ru
} 


\section{The algorithm of operational forecast of earthquakes}

The algorithm of the operational forecast of earthquakes, presented in this paper is based on a joint analysis of three parameters: the relative values of the deviations $\Delta f_{0} F_{2} / f_{\text {med }}$ of the critical frequency $f_{0} F_{2}$ from its median values $f_{\text {med }}$, the total values of the $K$-index for the day and the phases of the moon (Fig. 1). As conditions for declaring the beginning of the alarm period $t_{a l}$, the following criteria are selected: $\Delta f_{0} F_{2} / f_{\text {med }} \geq 0.2$ or the daily maximum of $f_{0} F_{2}$ falls within the interval 0-6 (UT), the sum of the K-index values for the day $\sum K \geq 20$, with the above two mentioned criteria in the intervals 7 days before and 7 days after the new moon phase. The duration of the alarm period is chosen equal $t_{a l}=5$ days.
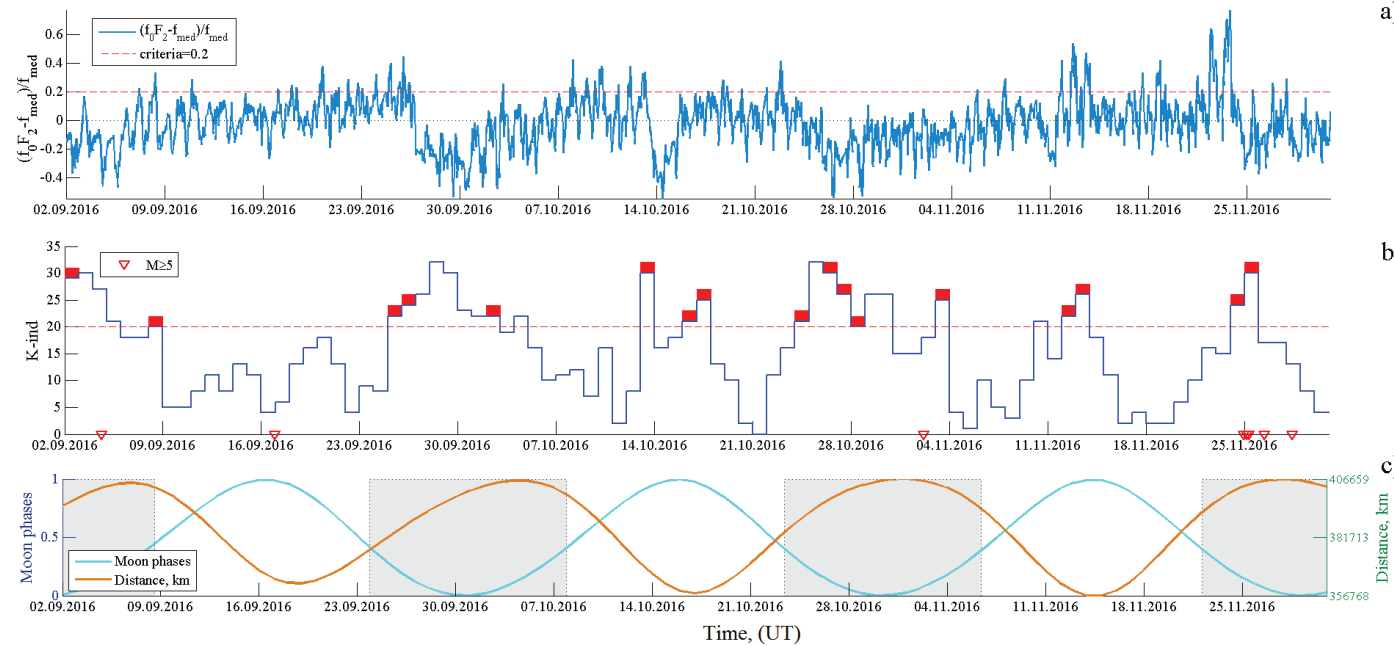

Figure 1. The temporal series of the the relative values of the deviations $\Delta f_{0} F_{2} / f_{\text {med }}$ (a). The temporal series of the daily values of the $K$-index (b). The temporal series of the values of the phases of the moon and its distances to the Earth (c). The red rectangles indicate the beginning of the alarm periods $t_{a l}$.

As predicted seismic events, earthquakes from the magnitudes $M \geq 5.0, M \geq 5.5, M \geq 6.0, M \geq$ 6.5 , occurred at depths of up to $100 \mathrm{~km}$ were considered. The radius of the earthquake preparation zone was determined according to the Dobrovolsky formula [2]

$$
\lg r=0.43 M
$$

and amounted to $140 \mathrm{~km}, 230 \mathrm{~km}, 380 \mathrm{~km}$ and $620 \mathrm{~km}$ for the considered ranges, respectively (Fig. 2) from the point of registration of ionospheric observations of IKIR FEB RAS in Kamchatka.

To analyze the efficiency of the considered precursor, the earthquake catalog of the Kamchatka branch of the Federal Research Center "United Geophysical Service of the RAS" [3] for 2009-2016 was used.

Reliability of the precursor $R$ was determined as the ratio of the number of earthquakes $n\left(E_{A}\right)$ for which the precursor was isolated to the number of all earthquakes $n(E)$ :

$$
R=n\left(E_{A}\right) / n(E)
$$




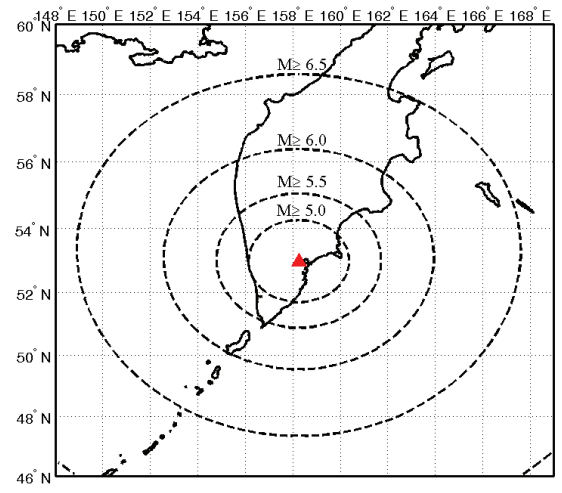

Figure 2. Areas of earthquake preparation with magnitudes $M \geq 5.0, M \geq 5.5, M \geq 6.0, M \geq 6.5$, determined by the Dobrovolsky radius.

The validity of the precursor $V$ was defined as the ratio of the number of predictive anomalies $n\left(A_{E}\right)$ to the total number of identified anomalies $n(A)$ :

$$
V=n\left(A_{E}\right) / n(A)
$$

Table 1. Dependence of the reliability and validity of the precursor from the lower threshold of the considered earthquake magnitudes.

\begin{tabular}{|c|c|c|c|c|}
\hline The predicted magnitude & $M \geq 5.0$ & $M \geq 5.5$ & $M \geq 6.0$ & $M \geq 6.5$ \\
\hline$r, \mathrm{~km}$ & 140 & 230 & 380 & 620 \\
\hline Reliability of the precursor $R$ & 0.28 & 0.11 & 0.38 & 0.625 \\
\hline Validity of the precursor $V$ & 0.057 & 0.049 & 0.065 & 0.04 \\
\hline
\end{tabular}

According to the results presented in Table 1, the reliability of the considered precursor is higher for earthquakes of higher magnitudes than for smaller ones. For earthquakes with a magnitude $M \geq 6.5$, the reliability $R$ was 0.625 . The validity of the precursor $V$ for all the magnitudes under consideration is low and its values do not exceed 0.065 . Since it is not uncommon for a precursor to be assigned several earthquakes (a group) and they need to be taken into account when deciding whether to implement a prediction and estimating the alarm time, the following algorithm was applied: 1) the forecast is considered to be the first earthquake that was implemented, corresponding to the forecast of the magnitude interval, 2) the alarm is removed, 3) the following earthquakes, which could formally correspond to the same predictive anomaly, are declared to be missed ("skipping the target") [4].

To evaluate the efficiency of the considered precursor, approaches by A.A. Gusev [5] and G.M. Molchan [6] were used.

Efficiency of the forecast according to A.A. Gusev's technique is calculated for a particular spatial region and a certain energy range of earthquakes according to the formula:

$$
J_{G}=\frac{N_{+} / T_{\text {alarm }}}{N / T}
$$

where $T$ is the total time of monitoring the seismic situation; $N_{+}-$the number of earthquakes that correspond to a successful prediction in time $T ; N$ - the total number of earthquakes that occurred 
(having spatio-temporal characteristics, similar to the predicted ones) that occurred during the time $T$; $T_{\text {alarm }}$ - total alarm time (the total duration of all time intervals in which the forecast for the estimated method operated during the total monitoring time). The statistical significance of $\alpha$ is determined by the probability of obtaining the efficiency values of $J_{G}$ in the absence of an earthquake-precursor relationship. In the absence of such a connection, i.e. when random guessing, the efficiency of $J_{G}$ is 1.

Table 2. Estimation of the efficiency of the precursor according to the A.A. Gusev method.

\begin{tabular}{|c|c|c|c|c|}
\hline The predicted magnitude & $M \geq 5.0$ & $M \geq 5.5$ & $M \geq 6.0$ & $M \geq 6.5$ \\
\hline$r, \mathrm{~km}$ & 140 & 230 & 380 & 620 \\
\hline$N_{+}$ & 7 & 6 & 8 & 5 \\
\hline$N$ & 25 & 51 & 21 & 8 \\
\hline$T$ (day) & 2922 & 2922 & 2922 & 2922 \\
\hline$T_{\text {expected }}$ (day) & 426 & 419 & 421 & 429 \\
\hline Efficiency $J_{G}$ & $1.97 \pm 0.72$ & $0.82 \pm 0.33$ & $2.64 \pm 0.93$ & $4.25 \pm 1.9$ \\
\hline$\alpha$ & 0.08 & 0.84 & 0.006 & 0.0025 \\
\hline
\end{tabular}

As can be seen from the results presented in Table 2, the efficiency of $J_{G}$ taking into account the standard deviation value assumes the largest values for the range of predicted magnitudes $M \geq$ 6.0 and $M \geq 6.5$. At the same time, the statistical significance of $\alpha$, which is 0.006 and 0.0025 , respectively, demonstrates a low probability of randomly obtaining such values in the absence of earthquake connection with the forerunner in question.

The efficiency of the precursor $J_{M}$ by the method of G.M. Molchan is defined as $J_{M}=1-v-\tau$, where $\tau=T_{\text {alarm }} / T$ - alarm measure; $v=1-N_{+} / N$ - the share of the misses of the target. For a random prediction $J_{M}=0$, and for the ideal (without a miss target and with zero alarm time) $J_{M}=1$.

Table 3 shows the values of $J_{M}$ for four values of the threshold magnitude of earthquakes $M \geq 5.0$, $M \geq 5.5, M \geq 6.0, M \geq 6.5$. For high magnitudes threshold ( $M \geq 6.5)$, the efficiency is substantially (almost 3.6 times) higher than for low $(M \geq 5.0)$.

Table 3. Estimation of the efficiency of the precursor according to the G.M.Molchan method.

\begin{tabular}{|c|c|c|c|c|}
\hline The predicted magnitude & $M \geq 5.0$ & $M \geq 5.5$ & $M \geq 6.0$ & $M \geq 6.5$ \\
\hline $\mathrm{r}, \mathrm{km}$ & 140 & 230 & 380 & 620 \\
\hline$\tau$ & 0.146 & 0.14 & 0.14 & 0.147 \\
\hline$v$ & 0.72 & 0.88 & 0.62 & 0.375 \\
\hline Efficiency $J_{M}$ & 0.13 & -0.025 & 0.237 & 0.478 \\
\hline
\end{tabular}

Figure 3 shows the Molchan diagram for the magnitude thresholds under consideration. On these diagrams, the abscissa of the point is defined as the anxiety measure $\tau$, and the ordinate is defined as the fraction of the passes of the target $v$. The diagonal of the Molchan diagram $\tau+v=1$, connecting the points $(0 ; 1)$ (the "optimist" point) and $(1 ; 0)$ (the "pessimist" point) corresponds to a random prediction. For this diagonal, it is possible to construct a confidence interval with a given significance $\alpha$. In this case, only the lower branch of this confidence interval is of interest. As follows from Fig. 3a and Fig. 3b, the values of $(\tau, v)$ obtained for magnitudes $M \geq 5.0$ and $M \geq 5.5$ lie above the lower limit of $99 \%$ of the confidence interval, which indicates the absence of a connection between the forerunner under consideration and earthquakes with given magnitudes that occurred on Distances of $140 \mathrm{~km}$ 
and $230 \mathrm{~km}$ from the observation point, respectively. The values $(\tau, v)$ obtained for earthquakes with magnitudes $M \geq 6.0$ (Fig. 3c) and $M \geq 6.5$ (Fig. 3d) lie below the obtained confidence interval boundary, which can be interpreted as a high degree of reliability of the revealed connection of the forerunner in question with earthquakes of this magnitude range.

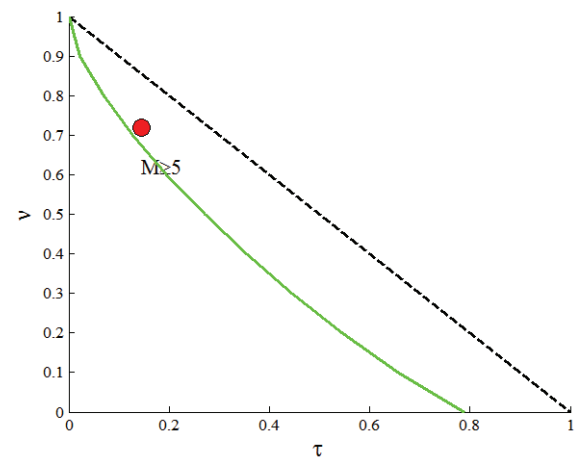

a)

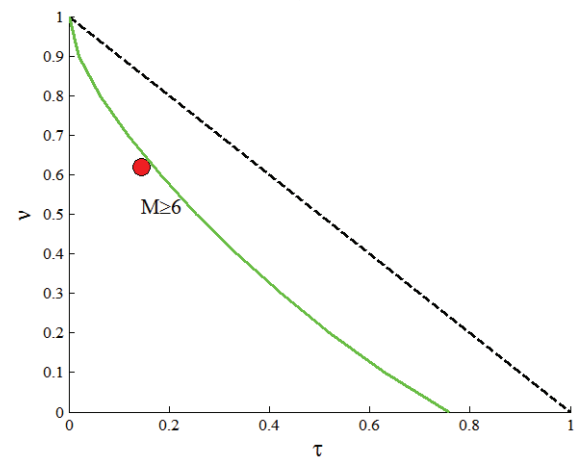

c)

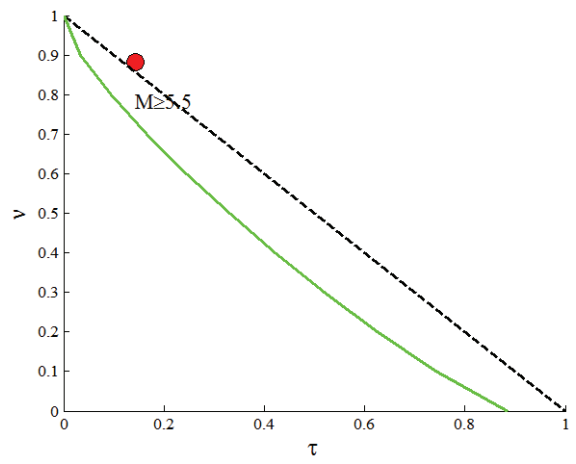

b)

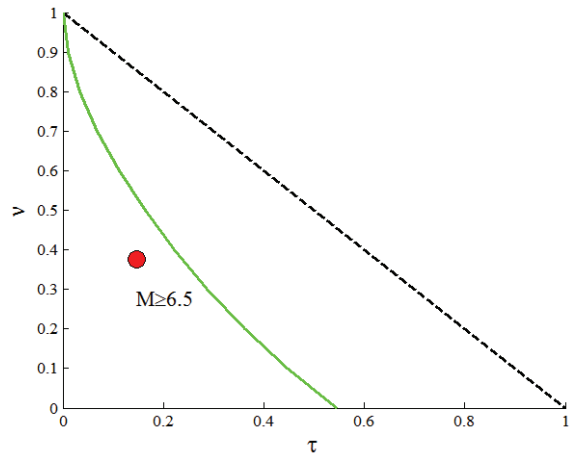

d)

Figure 3. Molchan diagrams for different thresholds of magnitude $M$. The lower bounds of the confidence interval of the random prognosis with the significance level $\alpha=0.01$ are noted.

\section{Conclusion}

An algorithm for the operational prediction of earthquakes is presented, in which the current values of the critical frequency $f_{0} F_{2}$ of the ionospheric layer $F_{2}$ exceed the median values during the periods of the disturbed state of the magnetosphere and the periods of the new moon as a precursor. For the predicted ranges of magnitudes $M \geq 5.0, M \geq 5.5, M \geq 6.0, M \geq 6.5$, the reliability $R$ and the reliability of $V$ have been calculated, and the efficiency of the precursor by the methods of A.A. Gusev and G.M. Molchan for the period 2009-2016. It is shown that for predicted earthquakes with a magnitude $M \geq 6.5$ the reliability of the precursor $R$ reaches the highest value and is 0.625 , and the reliability of $V$ for all considered magnitudes has low values and its value does not exceed 0.065 . The efficiency of $J_{G}$ by the A.A. Gusev assumes the values 2.64 and 4.25 for the ranges of the predicted magnitudes $M \geq 6.0$ and $M \geq 6.5$, respectively. In this case, the statistical significance of $\alpha$, which is 0.006 and 0.0025 , respectively, demonstrates a low probability of randomly obtaining such values 
in the absence of earthquake connection with the forerunner in question. The analysis of efficiency, conducted by the method of G.M. Molchan also shows a high degree of reliability of the revealed connection of the precursor in question with earthquakes from the magnitudes $M \geq 6.0$ and $M \geq 6.5$.

\section{References}

[1] V.V. Bogdanov, A.V. Buzevich, A.V. Vinitsky, G.I. Druzhin, A.V. Kuptsov, I.N. Poddelsky, S.E. Smirnov, B.M. Shevtsov, Complex seismological and geophysical research of Kamchatka. To the 25th anniversary of the Kamchatka Experimental-Methodical Seismological Department of the GS RAS (KEMSD GS RAS, Petropavlovsk-Kamchatsky, 2004) 259-278

[2] I.P. Dobrovolsky, S.I. Zubkov, V.I. Myachkin, Modeling earthquake precursors (Nauka, Moscow, 1980) 7-24

[3] http://www.emsd.ru/sdis/earthquake/catalogue/catalogue.php

[4] V.A. Saltykov, Physics of the Earth 2, 84-96 (2017)

[5] A.A. Gusev, Seismicity and seismic forecast, properties of the upper mantle and their relation to volcanism on Kamchatka (Nauka, Novosibirsk, 1974) 109-119

[6] G.M. Molchan, Physics of the Earth and Planetary Interiors 61, 84-98 (1990) 